\title{
Estudiu de les posibilidaes didáutiques de la traducción audiovisual (subtituláu y doblaxe) na enseñanza del asturianu n'Educación Primaria ${ }^{1}$ / Studying the didactic possibilities of audiovisual translation (subtitling and dubbing) in the teaching of Asturian in Primary Education
}

\author{
ROBERTO AVELLO-RODRÍGUEZ \\ ALBERTO FERNÁNDEZ-COSTALES \\ UNIVERSIDÁ D’UVIÉU
}

\begin{abstract}
Resume: Esti trabayu presenta datos empíricos d'un estudiu cuasi esperimental centráu n'analizar les posibilidaes didáutiques de la traducción audiovisual na enseñanza del asturianu. En concreto, esaminaráse la utilización del subtituláu y el doblaxe como ferramientes didáutiques na enseñanza de la llingua asturiana nel últimu ciclu d'Educación Primaria $\left(5^{\mathrm{u}}\right.$ y $\left.6^{\mathrm{u}}\right)$. Pal presente estudiu, contóse con una muestra de 62 alumnos d'Educación Primaria de 2 centros públicos del Principáu d'Asturies, qu'implementen una intervención docente na que los alumnos subtitularon y doblaron vídeos na clas de llingua asturiana durante 2 meses nel cursu académicu 2018-2019. Darréu, l'alumnáu participante contestó a un cuestionariu de rempuesta zarrada nel que se recoyíen les perceiciones de los escolares sobre l'usu del subtituláu y el doblaxe nel aula. Esti preséu compleméntase cola observación participante de los autores del estudiu nes aules de llingua asturiana. Los principales resultaos sorrayen les ventayes pedagóxiques del usu de la traducción audiovisual na enseñanza de la llingua tradicional asturiana, con una valoración bien favoratible per parte de la muestra participante, especialmente no que cinca al rodiu del usu del doblaxe. El trabayu conclúi apurriendo les implicaciones educatives que se deriven de la investigación en rellación cola enseñanza de la llingua tradicional d'Asturies.
\end{abstract}

Pallabres clave: traducción audiovisual, subtituláu, doblaxe, asturianu, Educación Primaria.

Abstract: The current paper reports on empirical data from a quasi-experimental research study delving into the didactic possibilities of audiovisual translation in the teaching of Asturian. Namely, the use of subtitling and dubbing as teaching resources in the teaching of Asturian Language in Primary Education ( $5^{\text {th }}$ and $6^{\text {th }}$ grades) is examined. The investigation relies on a sample of 62 students of Primary Education enrolled in 2 public schools in the Principality of Asturias which volunteer to implement a 2-month

\footnotetext{
${ }^{1}$ La presente investigación foi financiada pola Universidá d'Uviéu y la Conseyería d'Educación del Principáu d'Asturies, na «Convocatoria de Proyeutos d'Anovación ya Investigación Empobinaos al Personal Docente Investigador de la Universidá d'Uviéu 2019».
} 
intervention in the academic year 2018-2019.Participants answered a close-ended questionnaire enquiring about their perceptions on the use of subtitling and dubbing in the classroom. This tool was complemented with in-class observation by the authors of the study. The results of the investigation underline the pedagogic possibilities of audiovisual translation in the teaching of Asturian, with very favourable views reported by the students, especially as regards dubbing. The paper concludes by providing educational implications related with the teaching of the traditional language of Asturias.

Keywords: audiovisual translation, subtitling, dubbing, Asturian language, Primary Education.

\section{INTRODUCCIÓN}

La utilización del videu como recursu nel aula d'idiomes ye un tema afitáu na didáutica de les llingües, al amosase'l potencial y les ventayes del usu de materiales audiovisuales no que cinca al deprendizax del vocabulariu y la meyora na motivación del alumnáu (Vanderplank, 2019). Nel marcu actual, nel que los conteníos audiovisuales pasen a tener un papel esencial non solo na vida de la mocedá sinón tamién nel contestu educativu, ye imprescindible avanzar nes posibilidaes al rodiu l'usu de productos audiovisuales nel aula. Magar que la traducción audiovisual (TAV) ye un área afitada dientro los Estudios de Traducción y que tien un corpus relevante d'investigaciones sobre'l subtituláu, el doblaxe, la receición y l'accesibilidá, la llinia d'investigación qu'analiza'l papel del subtituláu y el doblaxe como téunica docente vien recibiendo una atención bien probe si consideramos el so potencial educativu. Nos caberos años, l'interés nesti ámbitu centróse n'esaminar el posible impautu de la producción de subtítulos y doblaxe per parte del alumnáu pa la meyora de la so competencia llingüística no que se noma como la vertiente educativa de la TAV (Incalcaterra, Lertola y Talaván, 2018).

La mayoría de trabayos espublizaos anguaño céntranse en midir l'efeutu que tien la TAV na formación de traductores, con estudios llevaos alantre solo nel contestu universitariu (Bolaños, 2017; Borghetti y Lertola, 2014; Ghia, 2012; Talaván, 2011 y 2019). Hai una falta bultable d'investigaciones que se propongan esaminar l'usu de la TAV nel ámbitu escolar y nun disponemos de resultaos sobre'l so posible impautu nes aules d'Educación Primaria o Secundaria (Fernández-Costales, 2017). Amás d'ello, la mayoría de trabayos centráronse en valorar el papel del subtituláu, con namái unos pocos artículos de recién inspeicionando l'usu del doblaxe nes aules (Sánchez-Requena, 2018; Talaván y Costal, 2017). D'últimes, la práutica totalidá de trabayos espublizaos hasta güei nel panorama nacional ya internacional lléndense na valoración del impautu que tien l'usu de la TAV na enseñanza del inglés como llingua estranxera.

El presente artículu tienta d'enllenar delles llagunes nesta llinia 
d'investigación al presentar datos empíricos algamaos nel contestu d'Educación Primaria, una etapa desaniciada hasta güei na lliteratura científica. Amás d'ello, la investigación nun se llenda namái n'estudiar l'efeutu del subtituláu o'l doblaxe de forma aisllada, sinón que conxuga les dos modalidaes nun mesmu proyeutu. Per último, la contribución más relevante de la investigación ye la d'abrir una nueva llinia al presentar, per primera vegada, un estudiu sobre l'usu de la TAV como recursu educativu na enseñanza del asturianu, un tema inéditu hasta la fecha. Los oxetivos de la investigación son los siguientes:

1) Analizar les perceiciones del alumnáu d'Educación Primaria sobre les posibles ventayes y dificultaes del usu de la traducción audiovisual nel deprendizax de llingua asturiana.

2) Esaminar les preferencies y apreciaciones del alumnáu sobre l'usu del subtituláu y el doblaxe nel deprendizax del asturianu nel contestu escolar. En casu d'identificase diferencies estadísticamente significatives ente dambes modalidaes, analizar les posibles causes.

3) Establecer les implicaciones pertinentes del uso de la TAV pa la enseñanza y el deprendizax del asturianu, teniendo en cuenta los resultaos algamaos.

\section{MARCU TEÓRICU}

Nesti apartáu referímonos a les tres pilastres fundamentales sobre les que se basa la presente investigación: l'usu de la TAV como recursu didáuticu na enseñanza de llingües; los trabayos previos espublizaos nos caberos años sobre l'usu del subtituláu y el doblaxe nel deprendizax de llingües; y los estudios disponibles sobre la metodoloxía y les anovaciones en materia d'enseñanza del asturianu n’Educación Primaria.

\section{LA TAV Y LA DIDÁUTICA DE LA LLINGUA}

Nes caberes décades, la TAV afitóse como un ámbitu d'investigación con entidá propia nos Estudios de Traducción. Dientro d'esta llinia, con resultaos granibles, la utilización del subtituláu - $\mathrm{y}$, en menor midida'l doblaxe- como recursu didáuticu foi analizáu dende dixebraes perspeutives valorando les posibilidaes que tienen dambes modalidaes nel deprendizax formal d'una llingua. ${ }^{2}$

\footnotetext{
${ }^{2}$ Ye importante señalar que nesti trabayu centrámonos namái na producción de subtítulos y doblaxe per parte del alumnáu pa la meyora de la competencia llingüística. L'usu de los subtítulos como recursu pasivu na enseñanza d'idiomes nun ye esamináu de forma esplícita, ya que -magar guarda una rellación evidente cola presente investigación- entrepasa los oxetivos d'esti trabayu, que ta llendáu a la producción activa de subtituláu y doblaxe.
} 
Ye importante alcordase de que l'usu de la traducción na enseñanza d'idiomes vien refugándose dende metanes el sieglu XX hasta anguaño, probablemente poles reminiscencies del métodu de Gramática-Traducción nel que l'alumnáu caltenía na memoria y repitía testos escritos pal deprendizax d'una llingua. Afitáu'l paradigma comunicativu na enseñanza de les llingües, tres los trabayos seminales de Hymes (1972), márcase l'entamu d'un periodu nel que la traducción desapaez nos métodos «nuevos» d'enseñanza de llingües (ver, por exemplu, Richards y Rodgers, 2014). De fechu, l'usu de la traducción nel aula rellaciónase frecuentemente con paradigmes anticuaos y poco centraos nel alumnu, magar qu'esisten estudios nos que se pon de manifiestu que la correuta esplotación y usu d'esti recursu nel aula puede apurrir ventayes pal alumnáu (Zabalbeascoa, 1990).

La publicación del Marcu Común Européu de Referencia pa les Llingües (Consejo de Europa, 2002) contribuyó en bona midida a la reconsideración del papel que puede xugar la traducción nel deprendizax de llingües al incluyila como una de les cuatro competencies básiques qu'establez el documentu: espresión, comprensión, interaición y mediación (o traducción). Amás, nel Marcu inclúyense dixebraes referencies al usu de la traducción pa la meyora de la competencia oral y escrita de los aprendices de llingües (pa un análisis en profundidá, encamiéntase revisar Talaván, 2013 o Lertola, 2018).

De manera más específica, y centrándonos na TAV, faise imprescindible citar l'estudiu pioneru nel nuestru país de Jorge Díaz-Cintas (1995), que yá defendía que'l subtituláu como téunica docente podía amestar variedá y meyorar la motivación na didáutica de les llingües, amás de tener un potencial nidiu sobre l'alquisición de vocabulariu nel alumnáu. Esti ye'l primer trabayu nel que se sofita de forma esplícita la producción de subtítulos per parte del alumnáu (y non namái'l visionáu de vídeos con subtítulos nel aula de llingua).

Darréu d'esti trabayu seminal, atopamos la monografía de Talaván (2013), qu'apurre la contribución más relevante fecha hasta anguaño nel nuestru país al asoleyar un modelu teóricu acompañáu d'unos encamientos práuticos pal usu del subtituláu como téunica docente nun aula de llingua. Esti trabayu toma como referentes el paradigma comunicativu y l'enfoque nociofuncional, asina como'l Deprendizax Basáu en Xeres p'allugar la utilización del subtituláu como téunica docente dientro de lo que se noma la dómina postmétodu na enseñanza de llingues (Talaván, 2013, p. 25). Amás d'ello, esamínense les posibles ventayes derivaes del usu de la subtitulación nel aula atendiendo a factores cognitivos y motivacionales, como la reducción de l'ansiedá nel aula en base a les teoríes de filtru afeutivu (Krashen, 1985). La contribución cimera de Talaván ye que presenta, per primera vegada, un análisis de la utilización del subtituláu nel aula d'idiomes so los parámetros non solo de los Estudios de Traducción, sinón teniendo en cuenta los modelos y paradigmes de la enseñanza d'idiomes, que permiten la efeutiva integración de la traducción 
audiovisual pa la meyora de la competencia llingüística del alumnáu.

Esta visión foi compartida apocayá por Lertola $(2018,2019 a)$ que fai un minuciosu repasu históricu pel papel de la traducción $-\mathrm{y}$, más específicamente, la traducción audiovisual- nes dixebraes dómines y corrientes na didáutica de la llingua y afonda nes ventayes de tipu afeutivu y motivacional derivaes del usu de la TAV. Los trabayos recién de Lertola tamién sofiten la posible integración de la TAV dientro de los enfoques comunicativos de la enseñanza de llingües, faciendo énfasis na enseñanza centrada nel alumnáu, y amplíen la perspeutiva al incluyir non solo'l subtituláu sinón tamién el doblaxe.

\section{INVESTIGACIONES PREVIES NEL USU DEL SUBTITULÁU Y EL DOBLAXE NEL DEPRENDIZAX DE LLINGÜES}

Na última década espublícense un interesante corpus d'investigaciones empobinaes a analizar les posibles ventayes del usu de la TAV n'estremaos contestos europeos, nos que se ratifica l'impautu que tien sobre l'alquisición y caltenimientu de vocabulariu (Danan, 2010; Lertola, 2019), la competencia intercultural (Borghetti y Lertola, 2014; Borguetti, 2011), la fluidez (SánchezRequena, 2013; Herrero, Sánchez-Requena y Escobar, 2018) o les competencies de producción oral y escrita (Talaván y Costal, 2017; Talaván, Rodríguez-Arancón y Martín-Monje, 2015). Les investigaciones feches hasta anguaño coinciden al señalar delles y nidies ventayes nel usu de la traducción audiovisual en dixebraos escenarios.

Nel casu del subtituláu, los estudios llevaos alantre hasta la fecha amuesen las virtúes que puede tener la producción activa de subtítulos pa la meyora de dixebraes dimensiones nel deprendizax d'una llingua estranxera. En concreto, asoléyense resultaos empíricos qu'avalen la meyora de la conciencia intercultural (Borghetti y Lertola, 2014), la capacidá pragmática (Incalcaterra, 2009), la comprensión oral del alumnáu (Talaván, 2011), la producción escrita de los estudiantes (Talaván y Rodríguez-Arancón, 2014), l'alquisición y caltenimientu de vocabulariu (Lertola, 2012), o la integración del subtituláu nel currículum de llingües estranxeres (Incalcaterra y Lertola, 2014).

Pela so parte, el doblaxe como recursu didáuticu vien recibiendo hasta güei una menor atención dende'l puntu de vista de la investigación (Talaván y ÁvilaCabrera, 2015). Les aveníes esploraes nesta llinia fueron de manera preferente la de la producción oral del alumnáu, especialmente no que cinca a la competencia fonética y la entonación (Kumai, 1996), asina como a la fluidez (Chiu, 2012; Danan, 2010). Más recién, los análisis céntrense na observación de la competencia oral del alumnáu de forma global con estudios holísticos y empobinaos a valorar l'impautu del doblaxe sobre la competencia comunicativa del alumnáu en rellación 
colos factores afeutivos (Talaván, Bárcena y Villarroel, 2014).

Les tendencies más nueves nesta llinia d'investigación tán marcaes polos trabayos nos que s'enancha l'espectru dientro l'estudiu de les posibilidaes didáutiques de la TAV pa ponderar la utilización del subtituláu pa sordos o persones con discapacidá auditiva (Talaván, 2019) o la audiodescripción (Moreno y Vermeulen, 2017), en dambos casos col oxetivu caberu de meyorar l'accesibilidá nel deprendizax d'una llingua.

Los resultaos algamaos hasta güei nesti campu son prometedores y sofiten el potencial de la TAV nel deprendizax de llingües. Sicasí, magar la tendencia al alza nel númberu d'investigaciones asoleyaes na última década, ye importante señalar delles debilidaes o llagunes qu'hai que tener en cuenta. Per un llau, la mayoría les investigaciones nesta llinia céntrense nel análisis d'una o otra modalidá de TAV, con mui pocos estudios que conxuguen les posibilidaes conxuntes del subtituláu y el doblaxe (Talaván y Ávila-Cabrera, 2015). Amás, les investigaciones sobre les posibilidaes didáutiques de la traducción suelen tomar como muestra alumnáu de los Graos n'Estudios de Traducción o de Llingües Modernes, y l'impautu que pueda tener la TAV nel contestu escolar siempre fue desaniciáu dafechu (Fernández-Costales, 2017). Otru de los aspeutos nos qu'hai entá un ampliu marxe de meyora refierse al diseñu d'investigación y rigor metodolóxicu de munchos estudios, que tienen en cuenta de forma xeneralizada muestres perpequeñes (con menos de 30 participantes nun gran númberu d'estudios). Per último, hai que sorrayar que, de forma mayoritaria, los trabayos nesta llinia d'investigación dedíquense a esaminar l'impautu de la TAV nel deprendizax d'una llingua estranxera (que, nesti casu, ye un sinónimu del inglés) y hai una falta nidia d'investigaciones que s'enfoten nel deprendizax d'una llingua minoritaria y/o minorizada. Nun s'atopen estudios sobre les posibilidaes pedagóxiques del subtituláu o'l doblaxe en llingües co-oficiales del Estáu Español -catalán, euskara o gallegu-, nin tampoco ye posible cuntar con resultaos empíricos en llingües minoritaries nel contestu européu. Poro, consideramos qu'esti trabayu ye pioneru nel sentíu de que puede abrir camín a otres investigaciones y a una llinia qu'esplore les posibilidaes que tien la producción activa de subtítulos y doblaxe nel deprendizax de la llingua tradicional d'Asturies.

\section{INVESTIGACIONES NA ENSENAANZA DEL ASTURIANU EN CONTESTOS EDUCATIVOS}

Hasta la fecha, nun ye posible atopar estudios sobre l'usu del subtituláu o'l doblaxe al rodiu'l deprendizax de la llingua asturiana, con namái una publicación centrada nesta última modalidá, na que Montero Domínguez (2016) esamina les carauterístiques propies de la traducción pal doblaxe n'asturianu. 
Per otru llau, tamién se quier dexar constancia de la falta d'estudios de tipu teóricu o empíricu nos que s'analicen cuestiones de calter didáuticu, pedagóxicu o educativu en rellación cola enseñanza del asturianu. Magar qu'esiste un corpus notable sobre la presencia del asturianu nel contestu escolar (González-Riaño y Fernández-Costales, 2019; Hevia-Artime y GonzálezRiaño, 2017; Bellido Calduch, 2018), les actitúes llingüístiques (Armesto Fernández, 2003; Dyzmann, 2000; González-Riaño, Hevia-Artime y Fernández-Costales, 2013), o la conocencia llingüística y el rendimientu del alumnáu escolarizao en llingua asturiana (Huguet y González-Riaño, 2002; González-Riaño y San Fabián, 1996), hai que señalar que nun abunden los trabayos qu'aborden -de manera específica-cuestiones metodolóxiques sobre la enseñanza y el deprendizax del asturianu nes etapes d'Educación Primaria y Secundaria, cola esceición del estudiu de González-Riaño y Armesto Fernández (2012) nel que se fai un repasu de tola xera didáutica d'esi profesoráu (dende la programación a la evaluación pasando pela metodoloxía...). ${ }^{3}$ Esta ye una cuestión sorprendente si tenemos en cuenta que ye una llinia d'investigación d'una evidente repercusión social y que podría valir p'apurrir datos empíricos que permitan, per un llau, analizar les posibles carencies y dificultaes de los mayestros (y que redundaría nuna meyora o optimización del procesu d'enseñanza y deprendizax de la llingua), y per otru llau, apurrir una radiografía basada en datos empíricos y que sirviríen d'encontu y sofitu na presencia del asturianu nes nuestres escueles.

Ente los trabayos qu'esploren de forma específica dalgún aspeutu rellacionáu cola enseñanza del asturianu n'Educación Primaria, ye importante destacar la investigación de Louzao y González-Riaño (2009) na que se ponía'1 focu sobre les actitúes llingüístiques ya interculturales del alumnáu llocal ya inmigrante de los tres ciclos d'esta etapa educativa. Los autores tomaron una muestra de 119 participantes y amosaron que les actitúes favoratibles escontra l'asturianu yeren compartíes tanto polos participantes llocales como polos estranxeros.

Magar nun ta averada al ámbito de la Educación Primaria, ye interesante citar equí la contribución recién de Suari (2020), qu'estudia les posibilidaes de l'alfabetización dixital pal caltenimientu de les llingües minoritaries. En concreto, esta investigación analiza los programas de formación virtual, centrándose nos procesos de desenvolvimientu, los componentes, los resultaos

3 Referímonos equí a investigaciones espublizaes en revistes científiques, tesis doctorales o informes qu'incluyan datos empíricos. La falta d'estes investigaciones nun suxer, en nengún casu, que nun esista una esmolición social por esta cuestión, nin que los docentes de llingua asturiana nun realicen les sos propies indagaciones nel aula al traviés del paradigma d'investigación-aición, anque estos esfuerzos nun cristalicen nuna publicación. 
educativos y los efeutos na normalización social del asturianu. Nuna investigación con un diseñu sólidu y rigurosu, que combina un exhaustivu trabayu etnográficu con un tratamientu estadísticu de los datos, Suari pon de manifiestu la trescendencia que tendrá l'emplegu de los medios audiovisuales y la formación a distancia nel futuru (adelantándose, de xuru, a lo qu'asocederá depués de 2020 y pon enriba la mesa la utilidá de los medios dixitales y la teunoloxía pa la supervivencia del asturianu). Nesti sen, esta investigación ta coneutada col presente estudiu, al resaltar el papel de los medios audiovisuales pa contribuyir a la revitalización de la llingua asturiana.

\section{EL PRESENTE TRABAYU}

Esti estudiu básase nun diseñu mestu siguiendo los enfoques plantegaos por Dörnyei (2009) en rellación cola investigación sobre'l deprendizax de llingües. En concreto, la investigación plantegada ye un estudiu cuasi esperimental onde los datos fueron recabaos direutamente de los participantes ${ }^{4}$, que siguieron una intervención didáutica de 8 selmanes de duración centrada na producción de vídeos subtitulaos y doblaos al asturianu en 2 centros del Principáu d'Asturies.

El proyeutu presentóse a los centros en xunetu de 2018 y solicitóse permisu pa comenzar la intervención col alumnáu nel mes d'ochobre. Organizóse un taller formativu col envís d'ufrir direutrices básiques sobre l'usu de la TAV a tolos docentes participantes, que tamién se formaron na utilización de les 2 ferramientes informátiques: Aegisub pal subtituláu y Windows Movie Maker pal doblaxe. Esti taller consistió nuna sesión formativa de 5 hores, distribuyíes de la siguiente forma: 1 hora pa subtituláu, 1 hora pa doblaxe, 1 hora pa la ferramienta de subtituláu, 1 hora pa la ferramienta de doblaxe y 1 hora pa les actividaes de dambes modalidaes. Confeicionóse una guía didáutica que s'entregó a los profesores y na que se incluyíen encamientos y direutrices didáutiques, información sobre’l subtituláu y el doblaxe asina como un conxuntu d'actividaes pa facer col alumnáu nes clases d'asturianu.

Los docentes trabayaron durante 2 meses coles actividaes (nes que los alumnos producíen los subtítulos y el doblaxe pa los vídeos) con un total de 8

\footnotetext{
${ }^{4} \mathrm{Na}$ presente investigación tuviéronse en cuenta tolos aspeutos éticos necesarios pal proyeutu y siguiéronse los estándares n'investigación en Ciencies Sociales y Xurídiques. Solicitóse autorización a la Conseyería d'Educación, Cultura y Deporte del Principáu d'Asturies, asina como a los centros participantes, qu'informaron al claustru y a les families sobre la naturaleza de la investigación. Tolos participantes accedieron a completar el cuestionariu de forma voluntaria y los datos de la investigación utilizáronse esclusivamente con envís académicu.
} 
hores. Les películes seleicionaes fueron Del Revés pal subtituláu y Harry Potter y la Piedra Filosofal pa les actividaes de doblaxe. Los vídeos consensuáronse colos docentes participantes y escoyéronse en función de la etapa educativa, los conteníos, y la rellación de les escenes seleicionaes colos usos llingüísticos de la etapa educativa na clase de llingua asturiana.

Les actividaes de subtituláu incluyíen subtituláu interllingual (Castellán $>$ Asturianu y Asturianu $>$ Castellán), xeres d'ordenación de subtítulos, exercicios de completar furacos en subtítulos, asina como xeres de creación de subtítulos alternativos, nes que l'alumnáu tenía qu'inventar diálogos nuevos pa los vídeos. En cuantes a les actividaes de doblaxe, realizáronse xeres de doblar a un solu personaxe, doblaxe de diálogos con varios personaxes (too ello n'asturianu), asina como de creación de diálogos alternativos. En tolos casos, los alumnos trabayaron en pareyes o en pequeños grupos pa favorecer la comunicación na llingua oxetu d'estudiu.

\section{Muestra}

La presente investigación tomó como muestra a 62 participantes de dos centros sosteníos con fondos públicos del Principáu d'Asturies: ún de los centros ta asitiáu nuna fastera urbana y l'otru nuna semi-urbana. Los dos centros respondieron afirmativamente a la convocatoria unviada polos autores del artículu nel marcu d'un proyeutu d'investigación financiáu pola Conseyería d'Educación y la Universidá d'Uvéu y focalizáu n'analizar les posibilidaes didáutiques de la traducción audiovisual. Buscábense centros qu'ufiertaren llingua asturiana n'Educación Primaria y que tuvieren interés en poner en prácutica una intervención nel aula. En concreto, analizáronse les perceiciones de 4 grupos d'alumnos de $5^{\mathrm{u}} \mathrm{y}$ $6^{\mathrm{u}}$ d'Educación Primaria con una media d'edá de 10,3 años. Los centros participantes taben allugaos na fastera central d'Asturies (Uviéu y Sama Llangréu) y accedieron a collaborar col proyeutu dientro d'una investigación más amplia centrada n'analizar les posibilidaes didáutiques de la TAV.

La carauterización de la muestra apaez reflexada na Tabla 1. Tolos participantes del estudiu nacieron n'Asturies, tán matriculaos na asignatura de Llingua Asturiana y confirmaron qu'usaben l'asturianu y el castellán de forma habitual na so vida diaria.

\begin{tabular}{|c|c|c|c|c|c|c|}
\hline & Homes & Muyeres & $5^{\mathrm{u}}$ & $6^{\mathrm{u}}$ & Asturianu & Castellán \\
\hline Sexu & $37,1 \%$ & $62,9 \%$ & & & & \\
\hline Cursu & & & $35 \%$ & $65 \%$ & & \\
\hline Llingua familiar & & & & & $30 \%$ & $70 \%$ \\
\hline
\end{tabular}

Tabla 1. Datos del alumnáu participante nel estudiu. 
El tipu de muestréu foi non probabilísticu (muestréu por conveniencia), ya que se requería que los centros participantes accedieren a que los mayestros punxeren en práutica la intervención didáutica de 8 selmanes de duración pa que l'alumnáu ficiera actividaes de subtituláu y doblaxe de vídeos nes clases d'asturianu. Los cuatro grupos esaminaos amosaben unes carauterístiques asemeyaes, cola única diferencia de la fastera na que s'atopa'l centru d'adscripción y les carauterístiques socio-económiques propies de caúna d'elles.

El tamañu muestral algamáu nun dexa realizar dellos análisis complexos típicos del tratamientu de datos con estadística inferencial, como l'análisis factorial, anque sí permitiría análisis de la varianza y análisis multivariantes que dexen una cierta significancia estadística teniendo en cuenta'l tipu d'estudiu realizáu (Dörnyei, 2014).

\section{INSTRUMENTOS}

L'instrumentu d'investigación utilizáu foi un cuestionariu de rempuesta zarrada. Los cuestionarios son la téunica de recoyida de datos más utilizada nesta llinia d'investigación y fueron emplegaos de forma consistente pa midir la eficacia del usu de la TAV nel deprendizax de llingües (Bolaños, 2017; Incalcaterra y Lertola; 2014; Sánchez-Requena, 2016; Talaván, 2019). Sicasí, nun esisten instrumentos previos que fueran específicamente diseñaos pa midir la perceición del alumnáu d'Educación Primaria sobre l'usu del subtituláu y el doblaxe, polo que s'ellaboró un cuestionariu ad hoc pal presente proyeutu, siguiendo les direutrices de los trataos más contrastaos sobre la construcción d'esti tipu d'instrumentos en didáutica de les llingües y deprendizax d'idiomes (ver, por exemplu, Brown, 2001; Dörnyei, 2014; Wilson y Dewaele, 2010).

El cuestionariu diseñáu taba compuestu d'una seición preliminar na que se recoyíen datos del alumnáu participante (en concreto, la edá, el sexu, el cursu académicu, la llingua familiar y el llugar de nacencia) y tres escales con 5 entrugues caúna (hasta completar un total de 15 ítems de rempuesta zarrada). Les dos primeres escales incluyíen les mesmes entrugues referíes, na primera seición al subtituláu y na segunda al doblaxe. En concreto, entrugábase al alumnáu sobre la so perceición sobre l'usu de les actividaes de TAV nel aula, les principales dificultaes atopaes, les actividaes más interesantes y la so opinión sobre la eficacia del subtituláu o'l doblaxe pa deprender asturianu. La tercera y última escala entrugaba sobre la preferencia d'una o otra modalidá de traducción audiovisual, cuál yera la más difícil de facer en clase y con cuál d'elles deprendieren más asturianu.

Les posibilidaes de rempuesta del alumnáu incluyíen una escala Likert de 5 puntos (siendo 1 - Totalmente en desalcuerdu; 2 - En desalcuerdu; 3 - Nin 
d'alcuerdu nin en desalcuerdu; 4 - D'alcuerdu; 5 - Totalmente d'alcuerdu), asina como rempuestes d'eleición múltiple pa los ítems sobre les actividaes feches nel aula y les dificultaes atopaes. Na última seición, los escolinos teníen que responder marcando una de les dos opciones (subtituláu o doblaxe). Teniendo en cuenta la edá de los participantes y el fechu de que les encuestes se ficieran nel aula (nel tiempu asignáu a l'asignatura de llingua asturiana), procuró ellaborase un instrumentu que pudiere ser contestáu de forma áxil y qu'en nengún casu sobrepasare los 15 minutos en total pa poder ser completáu por tolos alumnos.

L'instrumentu d'investigación sometióse a una valoración d'espertos, con tres docentes qu'evaluaron la idoneidá de les entrugues utilizaes. Amás d'ello, plantegóse una fase de pilotaxe con un grupu de 7 alumnos d'Educación Primaria que nun s'incluyó na muestra participante. Tres la validación y el pilotaxe, ficiéronse pequeños axustes en dellos ítems pa meyorar la consistencia interna del cuestionariu, que foi finalmente midida mediante l'Alfa de Cronbach $(\alpha=0,79)$ y demostró un bon nivel d'homoxeneidá interna.

Los cuestionarios pasáronse individualmente y en papel a lo cabero de la intervención didáutica nos centros n'avientu de 2018. Tolos participantes contestaron al cuestionariu de forma anónima y nel aula. Los datos recoyíos fueron procesaos pente medies del paquete estadísticu SPSS v. 24. Pa completar la información llograda colos cuestionarios, el proyeutu tamién incluyó observación participante nel aula, cola presencia de los autores del estudiu en delles sesiones de llingua asturiana. Les observaciones nel aula ficiéronse a la metá la intervención y a lo caberu'l proyeutu (selmanes 4 y 8 ) y taba focalizada n'esaminar cómo interactuaben los alumnos, les duldes que plantegaben al profesor, cómo yera la comunicación ente ellos y cuáles yeren les principales dificultades del alumnáu.

\section{ANÁlisis DE RESULTAOS}

A continuación, desglósense los principales resultaos collechaos del análisis estadísticu. Realizáronse análisis descriptivos de los datos (teniendo en cuenta les midíes, el rangu y la desviación típica) pa midir la perceición del alumnáu participante. Amás d'ello, utilizáronse test estadísticos non paramétricos (test del Chi-cuadráu y prueba de Kruskal-Wallis) puesto que la muestra del estudiu nun siguía una distribución normal.

Pa presentar los datos de forma más ordenada y sintética, y poder centranos nos oxetivos específicos del presente trabayu, referirémonos únicamente a los resultaos estrictamente rellacionaos cola perceición del alumnáu con respeuto al subtituláu y el doblaxe. En primer llugar, espónense 
los descriptivos más rellevantes rellacionaos cola primera de las tres escales de les que se componía'l cuestionariu utilizáu, centrada n'esplorar les perceiciones del alumnáu participante con respeuto al usu del subtituláu (Tabla 2):

\begin{tabular}{|c|c|c|}
\hline Escala 1 (subtituláu) & Media & Des. típica \\
\hline Prestóme subtitular vídeos en clase de llingua asturiana & 4,5161 &, 84430 \\
\hline Creo que deprendí asturianu subtitulando vídeos & 4,2419 &, 82354 \\
\hline
\end{tabular}

Tabla 2. Perceición del alumnáu sobre la realización de xeres de subtituláu en clase de llingua asturiana

En segundu llugar, referímonos a los resultaos de les apreciaciones del alumnáu sobre la realización de xeres de doblaxe nel aula (Tabla 3):

\begin{tabular}{|c|c|c|}
\hline Escala 2 (doblaxe) & Media & Des. típica \\
\hline Prestóme doblar vídeos en clase de llingua asturiana & 4,6129 &, 73227 \\
\hline Creo que deprendí asturianu doblando vídeos & 4,3710 &, 7320 \\
\hline
\end{tabular}

Tabla 3. Perceición del alumnáu sobre la realización de xeres de doblaxe en clase de llingua asturiana

A continuación, presentamos les preferencies de los alumnos encuestaos sobre la utilización del subtituláu y el doblaxe como téunica docente en clase de llingua asturiana (Gráficu 1):

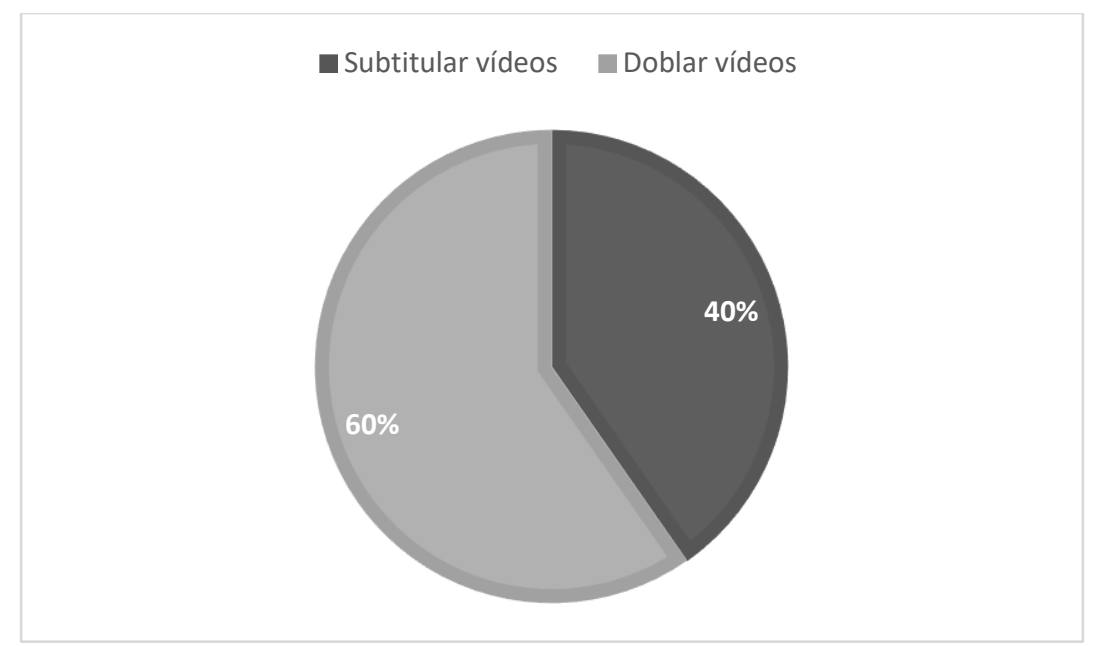

Gráficu 1. Preferencies del alumnáu participante sobre’l subtituláu y el doblaxe nel aula d'asturianu

La perceición de los escolinos participantes nel estudiu sobre l'efeutu que tienen el subtituláu y el doblaxe sobre’l deprendizaxe del asturianu apaez reflexáu nel Gráficu 2: 


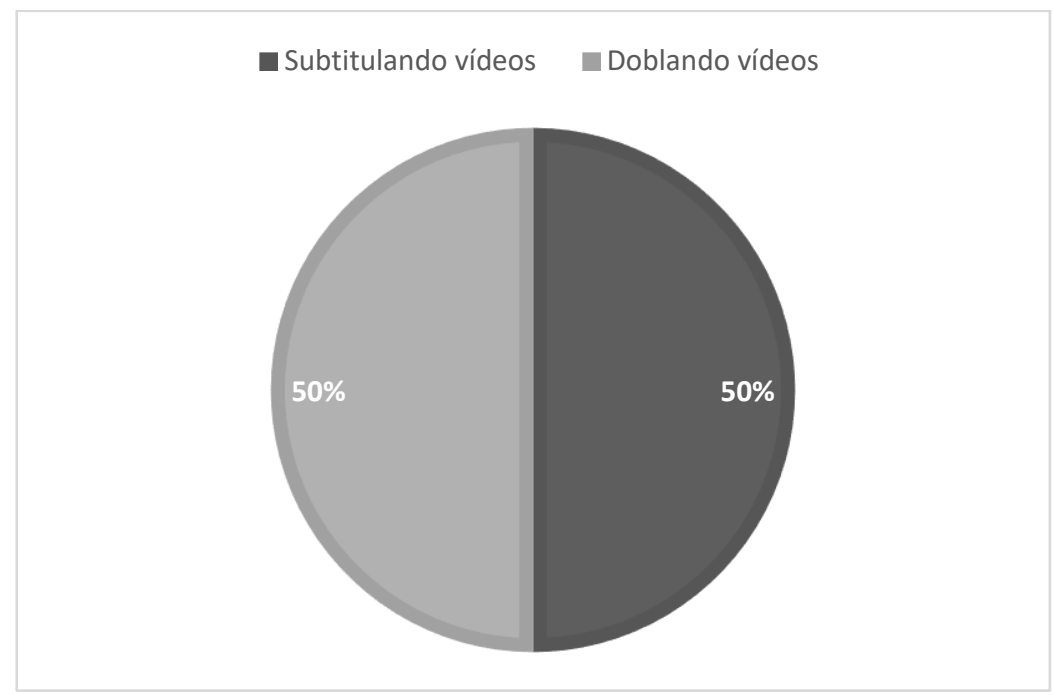

Gráficu 2. Resultaos del ítem 11 del cuestionariu:

¿Deprendisti más asturianu subtitulando o doblando vídeos?

A continuación, presentamos los resultaos de la valoración de los estudiantes sobre'l grau de dificultá asociáu al subtituláu y al doblaxe de vídeos en clase d'asturianu (Gráficu 3):

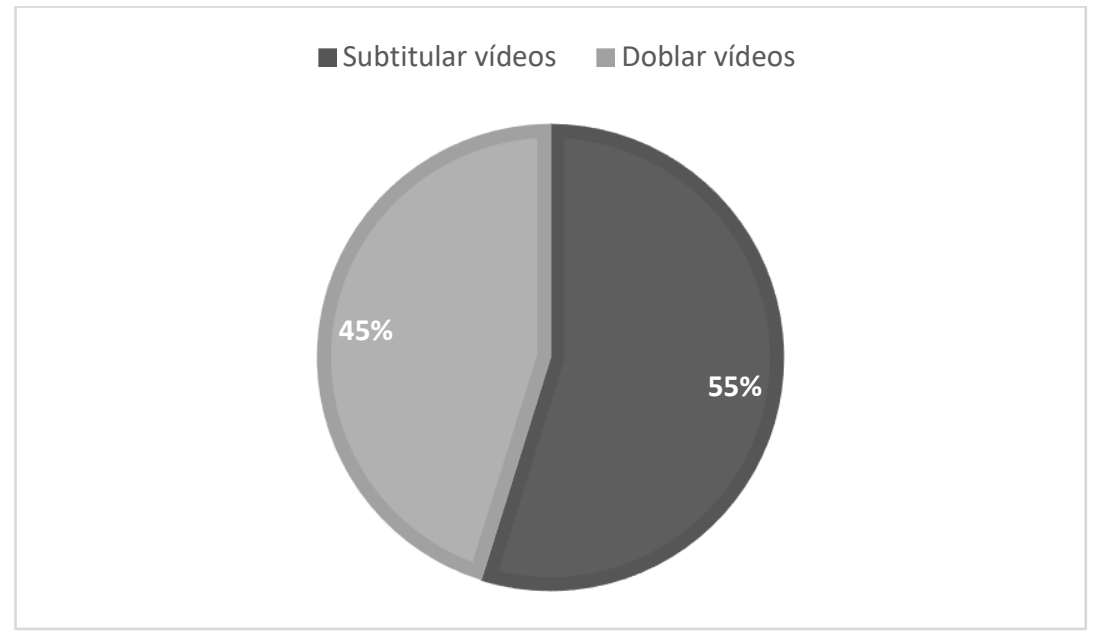

Gráficu 3. Dificultá del subtituláu o doblaxe de vídeos según los encuestaos.

Finalmente, los resultaos de los análisis non-paramétricos realizaos confirmen qu'hai diferencies estadísticamente significatives en función del xéneru de los participantes (Sig.=.000), lo que demostraría que les alumnes d'asturianu amuesen una meyor perceición tanto pa les actividaes de subtituláu como pa les de doblaxe, nun alcontrándose diferencies no que cinca a la tercera escala del cuestionariu (les preferencies sobre una o otra modalidá de TAV). 
Del mesmu mou, nun s'atopen diferencies estadísticamente significatives en nenguna de les tres escales en función del cursu de los participantes $\left(5^{\mathrm{u}} \circ 6^{\mathrm{u}}\right.$ de primaria), la edá o la llingua familiar.

\section{DISCUSIÓN}

Entamando col primer oxetivu del presente estudiu, podemos afirmar que los resultaos collechaos amuesen que l'alumnáu de llingua asturiana participante na investigación tien una actitú mui favoratible al rodiu les posibilidaes didáutiques de la TAV nel aula. En concreto, les dos modalidaes consideraes nel esperimentu tienen una acoyida perbona ente l'alumnáu d'Educación Primaria. Nesti sen, los resultaos coinciden coles investigaciones previes nel ámbitu de los Estudios de Traducción qu'analicen el potencial del subtituláu (Borguetti, 2011; Ghia, 2012; Lertola, 2016; López Cirugeda y Sánchez Ruiz, 2013; Talaván, 2006) $\mathrm{y}$-en menor midida, puesto que malapenes fueron esploraos- el doblaxe nel deprendizax d'una llingua (Danan, 2010; Talaván, Lertola, y Costal, 2016; Sánchez-Requena, 2018). Sicasí, ye necesariu incidir en que, de forma xeneralizada, los estudios referíos tuvieron centraos n'analizar la meyora de la competencia llingüística (alquisición de vocabulariu, meyora de la fluidez, habilidaes receutives, etc.) del alumnáu universitario nel deprendizax del inglés como llingua estranxera, y hai una falta nidia d'estudios realizaos nel contestu escolar (tanto n'Educación Secundaria como n'Educación Primaria). De manera más concreta, nun esisten investigaciones previes qu'aborden l'usu de la TAV nel procesu d'enseñanza y deprendizax del asturianu en nenguna etapa educativa.

Les perceiciones del alumnáu participante son perfavoratibles en rellación a les dos modalidaes de TAV esaminaes nel nuestru estudiu. Anque esisten ciertes diverxencies na valoración del alumnáu sobre les actividaes realizaes, les medies ( $x=4,61$ en caso del doblaxe y $x=4,51$ nel del subtituláu) confirmen que la valoración de dambes modalidaes ye perfavoratible, especialmente si tenemos en cuenta que'l sistema de rempuesta se puntuaba sobre 5. De fechu, hai que sorrayar que malapenes atopemos rempuestes negatives sobre l'usu de la TAV nel aula, col 82,9\% de los participantes afirmando esfrutar del subtituláu y un $92 \%$ amosando perceiciones favoratibles o mui favoratibles sobre'l doblaxe.

L'ampliu consensu nes rempuestes del alumnáu participante nun resulta sorprendente si tenemos en cuenta que toles actividaes propuestes nesti proyeutu impliquen un deprendizax centráu nel alumnáu y nel que los estudiantes lleven el pesu del procesu de deprendizax de la llingua, pente medies de la realización d'actividaes práutiques d'aula qu'impliquen el trabayu colos sos collacios (deprendizax collaborativu) y l'usu de les nueves 
teunoloxíes nel aula, dos pilastres fundamentales na enseñanza d'una llingua.

En cuantes a les dificultades atopaes, ye necesario apuntar equí que'l proyeutu requier tiempu y dedicación per parte del profesoráu que collaboró na investigación (con un taller previu y un total de 8 hores de docencia dedicaes a les actividaes de subtituláu y doblaxe). Amás, como asocede siempres que s'utiliza la teunoloxía nel aula, ye posible que se dean situaciones imprevistes qu'amenorguen la marcha de dalgún de los grupos. En cualesquier casu, ye conveniente tener en cuenta que siempres esisten alternatives analóxiques pa la realización del subtituláu y el doblaxe (por exemplu, ún de los grupos participantes optó por nun utilizar la ferramienta suxerida pal doblaxeWindows Movie Maker-y dexar que l'alumnáu lleera los diálogos creaos sobre'l propiu videu proyeutáu nel aula ensin utilizar nengún mediu nin programa informáticu adicional).

Siguiendo col segundu oxetivu del trabayu, ye necesario comentar que s'amuesa una cierta preferencia pol doblaxe con respeuto al alumnáu, probablemente porque los resultaos seyan más «visibles» cuando los estudiantes puen visionar un videu coles voces de los collacios, y esta modalidá permite-yos tamién prauticar con cuestiones prosódiques como la entonación. La observación participante nel aula permitiónos identificar una curiosidá nesti sen: los alumnos que - a priori- parecen más retrayíos nel aula o qu'intervienen menos de forma habitual (según la información proporcionada polos docentes) atopaben menos dificultaes pa participar nes actividaes de doblaxe. La nuestra hipótesis ye que, al tar el centru d'atención na pantalla y el visionáu del fragmentu de la película, esta situación permite-yos tar menos espuestos a les miraes de los collacios y rebaxa l'ansiedá nel aula, coneutando cola hipótesis del filtru afeutivu de Krashen (1985), que yá foi rellacionada col usu de la TAV (Lertola, 2018; Talaván, 2013) y del videu (Vanderplank, 2019) nel aula de llingua. Magar la llixera inclinación d'una mayoría de los participantes $(60 \%)$ escontra'l doblaxe de vídeos, nun s'aprecien diferencies estadísticamente significatives en cuantes al deprendizax del asturianu según la modalidá de TAV utilizada, y la dificultá llixeramente superior del doblaxe frente al subtituláu obedez -según la nuestra propia observación nel aula- al retu que supón la sincronización de los diálogos creaos pol alumnáu col videu.

Finalmente, hai que destacar que nel análisis de los datos nun s'amosaron diferencies estadísticamente significatives en función del cursu del alumnáu (toos ellos nel último ciclu d'Educación Primaria) nin de la llingua familiar. Sí s'atoparon diferencies en función del sexu del alumnáu, coles alumnes amosando una perceición más positiva de forma xeneralizada escontra l'usu y les posibilidaes didáutiques de la TAV nel aula, una conclusión que nun resulta sorprendente si tenemos en cuenta qu'esta ye una tendencia habitual nos estudios de deprendizax de llingües (ver, por exemplu, Pavlenko y Piller, 2008). 


\section{CONCLUSIONES}

La principal contribución d'esti artículu ye la presentación de resultaos d'una investigación empírica sobre l'usu de la TAV pa la meyora del procesu y deprendizax de la llingua asturiana. En concreto, pretendía apurrir datos que permitieren valorar la perceición del alumnáu de llingua asturiana n'Educación Primaria sobre les posibilidaes didáutiques de les dos principales modalidaes de TAV nel aula. Consideramos que'l presente trabayu realiza una contribución relevante por ser el primer estudiu qu'investiga l'usu pedagóxicu de la TAV pa la enseñanza de la llingua asturiana.

La perceición xeneralizada del alumnáu ye que la TAV tien cabida nes aules de llingua asturiana, si tenemos en cuenta la buena acoyida que tuvo tanto'l subtituláu como'l doblaxe. Anque ye posible atopar pequeñes diferencies ente dambes modalidaes, tanto una como otra son valoraes de manera mui favoratible polos participantes, que consideren que puen ser un bon recursu pal deprendizax de la llingua.

En segundu llugar, siguiendo coles implicaciones de la investigación, el presente estudiu ha sirvir como llamada pa siguir cola investigación no que cinca a la didáutica de la llingua asturiana y la metodoloxía de los docentes nel aula. Anque'l trabayu de los mayestros d'asturianu y el llabor qu'exercen nel aula ye bien conocíu, hai pocos trabayos nos que s'esamine qué ye lo que se ta faciendo nel aula, cuáles son les principales dificultaes metodolóxiques y cómo superales. Nesti sen, ye posible treslladar los diseños metodolóxicos y los tipos d'estudios que se faen anguaño con otres llingües de trabayu (inglés y castellán) al casu del asturianu, onde son escasos los acercamientos al usu de les nueves teunoloxíes nel aula. L'usu de la TAV ye un exemplu de cómo puede utilizase la teunoloxía pa facilitar el deprendizax de la llingua y enanchar les posibilidaes didáutiques y los recursos disponibles pa los mayestros. Tamién ye necesario resaltar que la conexón coles nueves teunoloxíes y, mui especialmente, colos productos audiovisuales, resulta un factor clave a esplorar nel ámbitu de la enseñanza y deprendizax del asturianu. Ye imprescindible da-y valir a la riqueza del asturianu al traviés de la Web y fomentar que los alumnos asturianos pescancien la importancia que tien l'usu de la llingua nos nuevos formatos pa garantizar el so caltenimientu. La subtitulación o'l doblaxe de material audiovisual (dexando a un llau'l so usu cómicu y les adautaciones virales que puen atopase n'Internet) puede facilitar l'accesu de munchos usuarios a materiales en llingua asturiana al traviés de comunidaes de fansubbing (subtituláu non profesional de series de televisión y películes) como yá asocede n'otros países y llingües minorizaes. Nesti sen, hai qu'acentuar el valor que tien la presencia de la llingua nos medios audiovisuales, que puen contribuyir a universalizar l'asturianu. 
Como en toles investigaciones, ye ineludible reconocer delles llimitaciones qu'han tenese en cuenta: l'artículu presenta un estudiu esploratoriu, que nun tien la vocación de ser estrapolable a tolos contestos y realidaes concretes de la enseñanza del asturianu. Coles mesmes, los resultaos, anque perfavoratibles, han tomase con cautela, ya que l'efeutu de deseabilidá y otros sesgos rellacionaos col emplegu de cuestionarios con alumnáu d'etapes escolares siempres puen tar presentes (ver Dörnyei, 2014). Tamién hai que reconocer que sería deseable poder cuntar con una muestra más amplia y representativa de la población oxetu d'estudiu -anque hai que matizar que resulta percomplicao algamar tamaños muestrales de muncha entidá nos estudios nos que resulta imprescindible una intervención didáutica nel aula a lo llargo d'un periodu prolongáu de tiempu, como ye'l casu del presente proyeutu-.

Finalmente, esti trabayu tien la vocación d'animar a que se realicen otres investigaciones centraes n'analizar el papel de la TAV nel deprendizax del asturianu: delles llinies a siguir a partir d'esti artículu puen ser la réplica del presente estudiu n'Educación Secundaria ya inclusive na universidá. Coles mesmes, podríen ampliase les modalidaes de TAV emplegaes, amestando audiodescripción pa meyorar l'accesibilidá nes aules. Nel apartáu metodolóxicu, sería conveniente cuntar con estudios llonxitudinales que se desarrollen durante un cursu académicu completu pa evaluar el posible efeutu cumulativu na utilización de la TAV nel deprendizax del alumnáu (Bisson et al., 2014; D’Ydewalle y Van De Poel, 1999). Dientro'l presente proyeutu d'investigación, yá se ta executando una segunda fase na que s'esaminarán les opiniones y perceiciones de los docentes involucraos pa cruzales colos datos collechaos de los alumnos, y va contase con un diseñu metodolóxicu de tipu mestu nel que les téuniques cualitatives de recoyida d'información completen los datos estadísticos equí presentaos.

Agradecimientos: los autores del presente trabayu quieren espresar de forma sincera la collaboración desinteresada, l'interés amosáu y tol trabayu realizáu polos dos centros escolares, los mayestros y l'alumnáu participante nesti estudiu. Por motivos de confidencialidá y en virtú de la política de proteición de datos, nun se revelen detalles o nomes específicos.

\section{BIBLIOGRAFÍA}

Armesto Fernández, X. (2003). Opiniones y actitúes de los estudiantes de Maxisteriu, Especialidá d'Educación Primaria: Estudiu de campu. Lletres Asturianes, 84, 65-84.

Bellido Calduch, T. (2018). Estudio de los niveles de uso, valoración y actitudes lingüísticas hacia la lengua asturiana que muestran los futuros maestros: Implicaciones educativas y curriculares. Tesis doctoral. Universidá d'Uviéu. 
Bisson, M. J., Van Heuven, W. J. B., Conklin, K., \& Tunney, R. J. (2014). Processing of native and foreign language subtitles in films: An eye tracking study. Applied Psycholinguistics, 35(2), 399-418. https://doi.org/10.1017/S0142716412000434

Bolaños García-Escribano, A. (2017). Reception and effects of fansubs on EFL education for translation and interpreting students: An empirical approach. Journal of Specialised Translation, 28(28), 122-163. https://www.jostrans.org/issue28/art_bolanos.pdf

Borghetti, C., \& Lertola, J. (2014). Interlingual subtitling for intercultural language education: A case study. Language and Intercultural Communication, 14(4), 423-440. https://doi.org/10.1080/14708477.2014.934380

Borguetti, C. (2011). Intercultural learning through subtitling: The cultural studies approach. En L. Incalcaterra McLoughlin, M. Biscio, \& M. À. Ní Mhainnín (Eds.), Audiovisual translation subtitles and subtitling: Theory and practice (pp. 111-137). Berna: Peter Lang.

Brown, J. D. (2001). Using surveys in language programs. Cambridge: Cambridge University Press.

Chiu, Y. H. (2012). Can film dubbing projects facilitate EFL learners' acquisition of English pronunciation? British Journal of Educational Technology, 43(1), 41-44. https://doi.org/10.1111/j.1467-8535.2011.01252.x

Consejo de Europa. (2002). Marco común europeo de referencia para las lenguas. Estrasburgo: Consejo de Europa.

D’Ydewalle, G., \& Van De Poel, M. (1999). Incidental foreign-language acquisition by children watching subtitled television programs. Journal of Psycholinguistic Research, 28, 227-244. https://doi.org/10.1023/A:1023202130625

Danan, M. (2010). Dubbing projects for the language learner: A framework for integrating audiovisual translation into task-based instruction. Computer Assisted Language Learning, 23(5), 441-456. https://doi.org/10.1080/09588221.2010.522528

Díaz-Cintas, J. (1995). El subtitulado como técnica docente. Vida Hispánica, 12(12), 10-14.

Dörnyei, Z. (2014). Questionnaires in second language research. Londres: Routledge.

Dyzmann, N. V. (2000). Averamientu al estudiu de contautu de llingües (asturianu / castellán) nel Principáu d'Asturies. Lletres Asturianes, 73, 93-105.

Fernández-Costales, A. (2017). Subtitling in CLIL: Promoting bilingual methodologies through audiovisual translation. En M. E. Gómez Parra \& R. Johnstone (Eds.), Educación bilingüe: tendencias educativas y conceptos claves = Bilingual educational: Trends and key concepts (pp. 185-196). Madrid: Ministerio de Educación. https://doi.org/10.4438/030-17-133-4

Ghia, E. (2012). Subtitling matters: New perspectives on subtitling and foreign language learning. Oxford: Peter Lang.

González-Riaño, X. A. \& Armesto Fernández, X. (2012). Enseñanza de la lengua minoritaria y la satisfacción del profesorado: el caso de Asturia. Cultura y Educación, 24(2), 219-241.

González-Riaño, X. A., \& Fernández-Costales, A. (2019). Principality of Asturias. En C. Lapresta-Rey \& Á. Huguet (Eds.), Multilingualism in European Language Education (pp. 53-71). Clevedon: Multilingual Matters. https://doi.org/10.9783/9781788923316-006

González-Riaño, X. A., Hevia Artime, I., \& Fernández-Costales, A. (2013). Language attitudes of Asturian students in the area of Navia-Eo (Spain). Language and Intercultural Communication, 13(4), 450-469. https://doi.org/http://dx.doi.org/10.1080/14708477.2013.785558

González-Riaño, X. A., \& San Fabián Maroto, J. L. (1996). La escolarización de la lengua asturiana. Su incidencia en el rendimiento educativo. Mieres: Editora del Norte.

Herrero, C., Sánchez-Requena, A., \& Escobar, M. (2018). Una propuesta triple. Análisis fílmico, traducción audiovisual y enseñanza de lenguas extranjeras. InTRAlinea Special Issue: Building Bridges between Film Studies and Translation Studies. http://www.intralinea.org/specials/article/2245

Hevia-Arrime, I., \& González-Riaño, X. A. (2017). Asturianu y castellanu nes aules d'Asturies: usu y actitúes del alumnáu inmigrante y autóctono. Lletres Asturianes, 116, 127-148.

Huguet, À., \& González-Riaño, X. A. (2002). Conocimiento lingüístico y rendimiento escolar. 
Implicaciones educativas en un contexto bilingüe. Infancia y Aprendizaje, 25(973), 53-68.

Hymes, D. (1972). On communicative competence. Sociolinguistics, 269293, 269-293. https://doi.org/10.1007/s11606-007-0364-4

Incalcaterra McLoughlin, L. (2009). Inter-semiotic translation in foreign language acquisition: The case of subtitles. En A. Witte, T. Harden, \& A. Ramos de Oliveira (Eds.), Translation in Second Language Learning and Teaching (pp. 227-244). Berna: Peter Lang.

Incalcaterra McLoughlin, L., \& Lertola, J. (2014). Audiovisual translation in second language acquisition. Integrating subtitling in the foreign-language curriculum. Interpreter and Translator Trainer, 8(1), 70-83. https://doi.org/10.1080/1750399X.2014.908558

Incalcaterra McLoughlin, L., Lertola, J., \& Talaván, N. (2018). Audiovisual translation in applied linguistics: Educational perspectives. Special Issue of Translation and Translanguaging in Multilingual Contexts, 4(1), 1-8. https://doi.org/10.1075/gest.8.3.02str

Krashen, S. (1985). The input hypothesis: Issues and implication. Londres: Longman.

Kumai, W. (1996). Karaoke movies: Dubbing movies for pronunciation. The Language Teacher Online, 20(9). https://jalt-publications.org/ttt/departments/myshare/articles/2049-karaokemovies-dubbing-movies-pronunciation

Lertola, J. (2012). The effect of subtitling task on vocabulary learning. En A. Pym \& D. OrregoCarmona (Eds.), Translation research projects 4 (pp. 61-70). Tarragona: Universitat Rovira i Visgili.

Lertola, J. (2016). Subtitling in language teaching: Suggestions for language teachers. En Y. Gambier, A. Caimi, \& C. Mariotti (Eds.), Subtitles and language learning: Principles, strategies and practical experiencesearning (pp. 245-267). https://doi.org/10.3726/978-30351-0719-7

Lertola, J. (2018). From translation to audiovisual translation in foreign language learning. Trans, $O(22), 185-202$. https://doi.org/10.24310/TRANS.2018.v0i22.3217

Lertola, J. (2019a). Audiovisual translation in the foreign language classroom: applications in the teaching of English and other foreign languages. https://doi.org/10.14705/rpnet.2019.27.9782490057252

Lertola, J. (2019b). Second language vocabulary learning through subtitling. Revista Española de Lingüistica Aplicada/Spanish Journal of Applied Linguistics, 32(2), 486-514. https://doi.org/10.1075/resla.17009.ler

López Cirugeda, I., \& Sánchez Ruiz, R. (2013). Subtitling as a didactic tool. A teacher training experience. Porta Linguarum, 20, 45-62.

Louzao Suárez, M., \& González-Riaño, X. A. (2009). Investigación sobre les actitúes interculturales del alumnáu asturianu d'Educación Primaria: un estudiu de casu. Lletres Asturianes, 100, 107-126.

Montero Domínguez, X. (2016). La traducción pal doblaxe n'asturianu. Un estudiu de casu. Lletres Asturianes, 114, 119-137.

Moreno, A. I., \& Vermeulen, A. (2017). Audio description for all: A literature review of its pedagogical values in foreign language teaching and learning. Encuentro, 26, 52-68. https://ebuah.uah.es/dspace/bitstream/handle/10017/34980/audio_ibañez_encuentro_2017_ N26.pdf? sequence $=1$ \&isAllowed=y

Pavlenko, A., \& Piller, I. (2008). Language education and gender. En N. H. Hornberger (Ed.), Encyclopedia of Language and Education (pp. 57-69). Nueva York: Springer.

Richards, J. C., \& Rodgers, T. S. (2014). Approaches and methods in language teaching. Cambridge: Cambridge University Press.

Sánchez-Requena, A. (2016). Audiovisual translation in teaching foreign languages: Contributions of revoicing to improve fluency and pronunciation in spontaneous conversations. Porta Linguarium, 26, 9-21.

Sánchez-Requena, A. (2018). Intralingual dubbing as a tool for developing speaking skills. Special Issue of Translation and Translanguaging in Multilingual Contexts. Audiovisual Translation in Applied Linguistics: Beyond Case Studies, 4(1), 102-128. 
Suari Rodrigue, C. (2020). L'enseñu virtual de la llingua asturiana (1996-2019): averamientu etnográficu, estudiu evaluativu y propuestes de futuru. Lletres Asturianes, 122, 165-196. https://doi.org/10.17811/llaa.122.2020.165-196

Talaván, N. (2006). Using subtitles to enhance foreign language learning. Porta Linguarum, 6, 41-52. http://www.ugr.es/ portalin/articulos/PL_numero6/talavan.pdf

Talaván, N. (2011). A quasi-experimental research project on subtitling and foreign language acquisition. En Laura Incalcaterra McLoughlin, M. Biscio, \& M. Á. Ní Mhainnín (Eds.), Subtitles and pubtitling. Theory and practice (pp. 197-218). Oxford: Peter Lang.

Talaván, N. (2013). La subtitulación en el aprendizaje de lenguas extranjeras. Madrid: Octaedro.

Talaván, N. (2019). Using subtitles for the deaf and hard of hearing as an innovative pedagogical tool in the language class. International Journal of English Studies, 19(1), 21-40. https://doi.org/10.6018/ijes.338671

Talaván, N., \& Ávila-Cabrera, J. J. (2015). First insights into the combination of dubbing and subtitling as L2 didactic tools. En Y. Gambier, A. Caimi, \& C. Mariotti (Eds.), Subtitles and language learning: Principles, strategies and practical experiences (pp. 149-172). Berna: Peter Lang.

Talaván, N., Bárcena, E., \& Villarroel, Á. (2014). Aprendizaje colaborativo asistido por ordenador para la transferencia de las competencias mediadora y lingüístico-comunicativa en inglés especializado. En M. L. Pérez Cañado \& J. Ráez Padilla (Eds.), Digital competence development in higher education: An international perspective (pp. 87-106). https://doi.org/10.3726/978-3-653-04536-9

Talaván, N., \& Costal, T. (2017). iDub - The potential of intralingual dubbing in foreign language learning: How to assess the task. Language Value, 9(1), 62-88. https://doi.org/dx.doi.org/10.6035/LanguageV.2017.9.4

Talaván, N., Lertola, J., \& Costal, T. (2016). iCap: Intralingual captioning for writing and vocabulary enhancement. Alicante Journal of English Studies, 29, 229-247.

Talaván, N., \& Rodríguez-Arancón, P. (2014). The use of reverse subtitling as an online collaborative language learning tool. Interpreter and Translator Trainer, 8(1), 84-101. https://doi.org/10.1080/1750399X.2014.908559

Talaván, N., Rodríguez-Arancón, P., \& Martín-Monje, E. (2015). The enhancement of speaking skills practice and assessment in an online environment. In M. L. Cancelas y Ouviña \& S. Sánchez Rodríguez (Eds.), Tendencias en educación y lingüistica (pp. 329-351). Cádiz: Editorial GEU.

Vanderplank, R. (2019). Video and informal language learning. En M. Dressman \& R. W.Sadler (Eds.), The handbook of informal language learning (pp. 181-201). Chichester: Wiley.

Wilson, R., \& Dewaele, J. M. (2010). The use of web questionnaires in second language acquisition and bilingualism research. Second Language Research, 26(1), 103-123. https://doi.org/10.1177/0267658309337640

Zabalbeascoa, P. (1990). Aplicaciones de la traducción a la enseñanaza de lenguas extranjeras. Sintagma, 2, 75-86.

Recibíu: 20.4 .2020

Aceutáu: 13.5.2020 